\title{
Beyond the usual suspects: A case study in choosing an inventory control system
}

Jennifer Watson*jennywatson@ou.edu, Cari Roberts cari.roberts@ou.edu

University of Oklahoma Libraries, 2601 Technology Place, Norman, OK 73071, United States of America

*Corresponding author.

\begin{abstract}
When the University of Oklahoma Libraries began planning a new high-density storage facility, we decided to look beyond the companies that had provided products specifically for libraries. We turned to records management companies, seeking out products used in the finance, insurance, healthcare, and legal industries. This column outlines our requirements and specifications, selection and implementation processes, and our current use of the software.
\end{abstract}

KeywordsInventory control systems; High-density storage; Library storage; Off-campus storage; Library software

\section{Introduction}

By volumes held, University of Oklahoma Libraries is the 32nd largest library in the United States, ${ }^{1}$ but is one of the latest ARL members to open a high-density storage facility. University of Oklahoma Libraries needed to catch up to this trend to accommodate remodels in both our main and branch libraries. The Library Service Center, or LSC, opened in September 2016 and was designed as both high-density storage and a new home for the Description and Access, Acquisitions, Discovery and Application Development, and Web Services departments. In less than 3 years, this facility has reached over $90 \%$ capacity.

The LSC was designed to use the Harvard Model, ${ }^{2}$ shelving items by size rather than classification, and to hold 500,000 low-use items. Since the facility was a remodel of an existing building, once used as a call center and reimagined as a shared work and storage space, the shelves are only 10 ' tall, with the building

temperature and humidity set to accommodate people rather than adhering to archival best practices. Our previous off-site storage facilities were nearing capacity and used traditional library shelving with items 
organized either by call number or in barcode number order. We needed a stronger and more flexible organizational system and looked to our colleagues at the Oklahoma State University Library Annex Building and other high-density storage facilities for inspiration.

After viewing a few inventory control systems in action, we decided to look beyond the companies that had provided products specifically for libraries. Products we saw at other facilities were dated; we needed something that would operate similarly to the software products student workers were familiar with and less like MS-DOS. We turned to records management companies, seeking out products used in the finance, insurance, healthcare, and legal industries.

\section{Requirements and specifications}

Before choosing a product, we outlined what our current workflows looked like, what our ideal workflow looked like, and our technical specifications. Products needed to meet the following objectives:

- Tracks current inventory and location by tray and shelf through barcoding

- Makes use of existing 12 digit barcodes that use prefix and sequence validation

- Tracks inventory usage

- Provides library staff with the ability to run inventory reports.

These objectives were outlined in a request for proposal (RFP) along with more general requirements about training, documentation, and maintenance and support. The RFP also outlined the kind of information we would need about hardware requirements and equipment recommendations:

- Product hosting

$\circ$ Vendor and/or OU

○ Server type

○ Operating system

○ Storage space needed

- Equipment recommendations

- Barcode scanners

○ Barcode printers

- Any other equipment recommendations

The proposal period lasted 4 weeks.

\section{Selecting an inventory control system}

Once all proposals were received, we evaluated them on software usability, compatibility with our library service platform Ex Libris 's Alma, hardware and equipment requirements, detail level of reporting functions, 
the implementation plan and schedule, maintenance and support, cost, and the company 's experience. ${ }^{3}$ After asking three companies to demonstrate their products via video conference, and contacting client references, we were most impressed by Zasio 's Versatile Professional (VP) ${ }^{4}$ Versatile Professional was the most ready to go out of the box. The program 's print documentation and in-software help functions, and the option to use the MSSQL Server platform to easily import data from Alma, were key benefits. Though the initial cost of VP was not the lowest among the three demonstrated products, the other vendors' customer support and maintenance costs were separate. These would have quickly accrued and might have been necessary several times over the lifetime of the product.

\section{Implementation processes}

As part of our acquisition of Versatile Professional, Zasio sent a trainer to provide $12 \mathrm{~h}$ of on-site training for our hands-on users. We were provided with well written, clearly organized training manuals as well as live demonstrations on a test database, based on a subset of our collection. Using our own data let us identify potential pitfalls and introduced Zasio staff to issues unique to academic libraries. Zasio had worked with records centers and one archive before, so we needed to familiarize them with library terminology and metadata standards.

Having received the training, manuals, and installation software from Zasio, we hit the ground running. We spun up a virtual server and installed the MSSQL Server platform. The documentation clearly outlined the SQL database configurations. Once that was in place, we installed the VP application on designated computers. For network security reasons, we chose not to use the application over the wireless network.

We then worked with our Zasio software support specialist to set up appropriate fields in the database. Approximately one-third of our implementation time was spent on this task. Being a library, not a warehouse, we have special needs related to the specific fields and information we require for inventory control. These customized fields facilitate retrieval and accurate reporting. We also had to keep in mind scalability and the types of items to be held, as we plan to increase the size of our facility and the variety of items stored within it in the near future.

In order to make this all work, scripts were written to facilitate API calls in Alma. These calls brought in the data that populates the customized fields. With API calls underway, we held many conversations with our Zasio software support specialist in order to properly map the fields and also to set up the import process. We created a representative set of the types of items we would be importing and conducted a test import. After a few tweaks to the import process and scripts, we were able to successfully import our first set of records into the test database, and after additional testing, the test database became our production database.

The original estimate of implementation was 40 days. Our active time was closer to 20 days, and our actual time was closer to 3 months. Progress was delayed due to a wait for campus IT changes to our server, scheduling in-person training, and our winter academic break.

\section{Post-implementation processes}


With implementation complete, we have a process in place for importing records, as we know what items are coming into the Library Service Center. We use either Alma queries or physical barcode scanners to obtain a list of barcodes. This barcode list is run through a series of Alma API calls that result in a file that can be imported into the database. Once item records have been created in the database, the physical item can be scanned as it arrives at the LSC to be boxed, and all of the appropriate fields are already populated in the Versatile Professional application. There is some manual work involved, but it 's a rather efficient process; at the peak of our ingestion processes we were bringing in up to 5000 items per week.

Another process we developed creates a daily transfer report that is emailed to the Metadata Maintenance unit. Versatile Notification System, a subroutine within Versatile Professional, runs a query in the background which triggers a notification, and an email with an attached Excel document is sent. Included in the report are the barcode, title, call number, description, MMS ID (Alma 's unique identifier for records), event date, and shelving location. This allows Metadata Maintenance unit personnel to change the item 's location in Alma, but also provides enough information that they can pull items they need to enhance, or correct item and holdings records.

Once items are ingested and their locations are updated, library users can request them through a link in Primo, our discovery layer. This link directs the user to a document delivery form that is processed by the Interlibrary Loan department. In Alma, ILL staff place physical item requests on materials associated with document delivery and interlibrary loan requests. We developed a daily process that uses a series of Alma API calls to acquire a list of all items that need to be collected from various storage locations. Once the list is obtained, it is imported into the database as a table, and a set of queries are run against it to retrieve a box number and location for those items. Without this process, each item would have to be looked up in Alma, and then again in Zasio, in order to find out where it's stored. This is a big time-saver because of the number of requests we get for items held at the Library Service Center.

\section{Truly versatile}

Versatile Professional's flexibility has allowed us to expand its usage into areas we had not initially considered. One is our Special Collections. The on-campus home for our Western History Collections is overcrowded and as a result, some low-use manuscript collections have been moved to a secure area of the Library Service Center. VP offers the ability to create new box types and storage rooms, so we were able to create document case, cubic foot, and flat case box types to reflect the storage requirements of special collections materials, and should the need arise, we will be able to accommodate artifacts and artwork, as well. Since many of these special collections items have been cataloged only at the collection level, adding them to Versatile Professional gives Special Collections personnel the opportunity to catalog at the item or box level.

A feature that we have found very useful is the many ways we can edit records. If the Metadata Maintenance unit identifies an error in the item or holding record as they perform a transfer, they can notify LSC staff and the record can be edited manually. If a change needs to be applied to multiple records, we have the ability to conduct a query and then write script to update the appropriate records directly in the database. We have also created queries to look for boxes and records with incorrect information. For example, we accidentally created multiple boxes for a single shelf position because we had not assigned one of the boxes to a storage room. 
These orphan boxes were identified and corrected through the use of a custom query. Access to the database makes it possible to perform many custom queries as needs arise.

We have also expanded our use of Versatile Professional to accommodate oversize books and artifacts. We needed dedicated space for oversize items being transferred from our Fine Arts and Geology branch libraries into the LSC, so we created a new box type called Unboxed for these items. Included in these unboxed areas are a camel saddle, books as large as $3 \quad$ ' $\quad$ x $55^{\prime}$, and framed art that are stored directly on the shelf with acid-free labels attached with unbleached cotton tying tape.

We 're also considering using the system for our off-site maps and microform systems. Since these collections contain items in Dewey, Library of Congress, Superintendent of Documents, and numbered systems, conceptualizing the cabinets and drawers as bays and shelves will help student workers and staff find items more quickly.

\section{Conclusion}

Although Versatile Professional was not created with the library market in mind, we have been very pleased with it. Successfully operating a high-density storage facility is, essentially, a matter of inventory control, so selecting and adapting an inventory control system to meet library storage needs makes good sense. By moving beyond the two paths generally pursued by libraries for this purpose-either utilizing the most common system in place at high-density library facilities, which is not particularly intuitive or competitively priced, or creating an in-house system, which requires significant local resource investment-we were able to select a product which was reasonably priced, highly flexible, easy to use, and capable of growing to meet our needs.

\section{Footnotes}

[1] The Nation's Largest Libraries: A Listing By Volumes Held. (2014, August 26). Retrieved October 18, 2019, from http://www.ala.org/tools/libfactsheets/alalibraryfactsheet22.

[2] David Weeks MLS \& Ron Chepesiuk MLS, DAS (2003) The Harvard Model and the Rise of Shared Storage Facilities, Resource Sharing \& Information Networks, 16:2, 159-168, DOI: $\underline{\mathrm{ht}}$ tps://doi.org/10.1300/J121v16n02 03

[3] Both the RFP (https://hdl.handle.net/11244/323807)and evaluation rubric (https://hdl.handle.net/11244/323806) are located in our institutional repository at $\underline{\text { SHAROK.o }} \underline{\text { rg. }}$

[4] Versatile Professional. Retrieved December 19, 2019 from https://www.zasio.com/technology -solutions/records-management-software/versatile-professional/ 